\title{
Intervenciones eficaces vía internet para la salud emocional en adolescentes: una propuesta ante la pandemia por COVID-19
} \author{
pandemic COVID-19 \\ Anabel de la Rosa Gómez ${ }^{1}$ \\ https://orcid.org/0000-0002-3527-1500 \\ Liliana Moreyra Jiménez ${ }^{2}$ \\ https://orcid.org/0000-0002-8123-6589 \\ Nayeli Guadalupe de la Rosa Montealvo ${ }^{3}$ \\ https://orcid.org/0000-0002-3833-4669 \\ Universidad Nacional Autónoma de México
}

Effective online interventions for emotional health in teenagers: a proposal to

Recibido: $15-06-2020$

Aceptado: 03-08-2020

\section{Cita Recomendada}

De la Rosa, A., Moreyra, L. \& De la Rosa, N. (2020). Intervenciones eficacies vía Internet para la salud emocional en adolescentes: Una propuesta ante la pandemia por COVID-19. Hamut'ay, 7 (2), 18-33.

http://dx.doi.org/10.21503/hamu.v7i2.2128

\section{RESUMEN}

El objetivo del presente estudio fue realizar una revisión de las intervenciones eficaces vía internet para la salud emocional en los adolescentes y presentar una propuesta de intervención breve basada en técnicas de psicología positiva para potenciar la resiliencia ante la pandemia por COVID-19. Para ello se realizó una búsqueda de artículos publicados sobre intervenciones eficaces vía internet para la salud emocional en los adolescentes entre el año 2010 al 2020 en las bases de datos PsycINFO, Scielo, PubMed y Google Scholar. Se encontraron 13 estudios científicos de intervenciones a través de internet o teléfono móvil con resultados alentadores en población adolescente de entre 10 a 19 años con problemas emocionales o para la prevención de la salud mental. Además, se realizó una propuesta de intervención breve que consiste en un programa autoaplicado a través de internet integrado por 12 componentes terapéuticos basados en técnicas en psicología positiva. Los programas autoaplicados se caracterizan por ser autoadministrables y funcionan sin apoyo de un terapeuta en tiempo real, permite que la terapia llegue a todos aquellos que la necesiten sin tener que salir de casa, proporciona una mayor flexibilidad en cuanto al acceso y tiempo del tratamiento, así como una disminución del costo de la terapia. Es necesario el desarrollo de programas de intervención en psicología basados en la evidencia que favorezcan la disminución del impacto psicológico durante las diferentes fases de la epidemia por COVID-19 para el bienestar de la población, principalmente

\footnotetext{
1 Doctora en Psicología. Profesora de carrera titular A, tiempo completo, Facultad de Estudios Superiores Iztacala. Responsable académica del Laboratorio de Psicología e Innovación Tecnológica (LABPSIIT). anabel.delarosa@iztacala.unam.mx

2 Licenciada en Psicología. Profesora de Asignatura A. Miembro del Laboratorio de Psicología e Innovación Tecnológica (LABPSIIT). liliana.moreyra@iztacala.unam.mx

3 Licenciada en Psicología. Miembro del Laboratorio de Psicología e Innovación Tecnológica (LABPSIIT). nayeli_delarosa@ired.unam.mx
} 
en la población vulnerable como los niños y los adolescentes.

Palabras clave: intervenciones eficaces, internet, adolescentes, COVID-19, resiliencia, psicología positiva.

\begin{abstract}
The study aimed of this reviewed was to undertake of the effective online interventions for emotional health in adolescents and bringing forward a proposal shortly intervention based on psychology positive techniques to strengthen resilience to Pandemic COVID-19. For this purpose, a searchable article published on effective online interventions for emotional health in adolescents between 2010 to 2020 in the PsycINFO, Scielo, PubMed y Google Scholar. Databases. Thirteen scientific interventions studies were found by internet or mobile phone with encouraging results in the adolescent population between 10 to 19 years with behavioral problems or for mental health prevention Additionally, a proposal shortly intervention was drafted that consisting on a self-administered program on the internet comprised for 12 therapeutic compounds based on positive psychology techniques. The self-administered programmers are characterized to be self-administrative and function without support of a therapist in real time, the therapy allows to reach all those people who need it, without having to leave their homes, provides more flexibility on access and treatment time, as well as a low cost of the therapy. Special attention of program development of intervention in psychology on set evidenced based to favor the reduction of psychological impact during the different phases of the epidemic por COVID-19 for the improvement of people's well-being particular vulnerable populations as children and teenagers.
\end{abstract}

Keywords: effective interventions, online, teenagers, COVID-19, positive psychology, resilience

\section{INTRODUCCIÓN}

El brote del nuevo Síndrome Respiratorio Agudo por Coronavirus 2 (SARS-CoV-2) se ha convertido en una emergencia de salud pública de preocupación internacional que no solo ha amenazado la salud física, si no también ha afectado la salud mental y el bienestar psicológico de las personas.

Según un estudio realizado para evaluar el impacto en la salud mental de la población en la fase inicial del brote de SARS-CoV-2, más de la mitad de los encuestados obtuvieron resultados negativos entre los niveles moderado y severo. Asimismo, los participantes reportaron síntomas depresivos (16.5\%), ansiedad $(28,8 \%)$ y niveles de estrés moderados a severos (8.1\%) (Wang et al., 2020). Posteriormente, otro estudio encontró un aumento en las emociones negativas (ansiedad, depresión y enojo) y sensibili- dad a los riesgos sociales, así como una disminución de las emociones positivas y satisfacción con la vida después de la declaración oficial como epidemia del COVID-19 en China (Li et al., 2020).

Se ha enfrentado a la pandemia del COVID-19 con medidas de salud pública para mitigar la propagación del virus, tales como el distanciamiento físico y el confinamiento en países de todo el mundo. Sin embargo, pese a que estas medidas pueden ser críticas para mitigar la propagación de esta enfermedad, la separación de los familiares y amigos, la percepción de pérdida de libertad y la incertidumbre de su evolución, en ocasiones, resulta en efectos psicológicos adversos.

$\mathrm{Al}$ respecto, Brooks et al. (2000) indagaron el impacto psicológico durante la contingencia sanitaria y encontraron que el confinamiento, los cambios 
en la rutina habitual y la reducción del contacto social con los demás provocaban aburrimiento, frustración y aislamiento, lo cual era angustiante para los participantes. En su mayoría se encontraron efectos psicológicos negativos, tales como síntomas de estrés postraumático, depresión, ansiedad, confusión y enojo Alvites-Huamaní, (2020). Los predictores del impacto psicológico fueron una mayor duración de confinamiento, tedio, miedo a las infecciones, frustración, falta de suministros básicos, pérdida de confianza en la información, problemas económicos y estigma social.

Ahora bien, la adolescencia es una etapa de gran relevancia para la consolidación de estilos de vida que permitan mantener la salud y el bienestar, sin embargo, al mismo tiempo es un periodo de alta vulnerabilidad debido a las conductas de riesgo que determinan su salud física, emocional y mental (Valenzuela, et al., 2013; Alvites-Huamaní, (2019); tales como los accidentes, suicidios, violencia, complicaciones relacionadas con el embarazo, entre otras (Corona \& Peralta, 2010).

Ante este panorama, es importante formular estrategias de intervención innovadoras y accesibles para reducir los impactos psicológicos adversos durante y después de la pandemia en la población de mayor vulnerabilidad, como lo son los adolescentes.

Por otro lado, en los últimos años se han incorporado con éxito y de manera exponencial las Tecnologías de la Información y Comunicación (TIC) a las intervenciones psicológicas. Se han desarrollado intervenciones que pueden ser administradas a través de Internet capaces de reducir el tiempo de contacto entre paciente y terapeuta y de llegar a personas que de otra forma no recibirían tratamiento (Andersson, 2016). Diversos estudios respaldan la evidencia sobre la eficacia de las intervenciones apoyadas por Internet (Andrews et al., 2010; Cárdenas, Botella, Quero, De la Rosa \& Baños, 2014; Ebert et al., 2018; Herrero et al., 2018; Pasarelu, Andersson, Bergman \& Dobrean, 2017).

\section{Telepsicoterapia}

Las TIC se define como aquellos recursos y dispositivos tecnológicos que permiten la producción, gestión y diseminación de información a través de diversas redes, tales como la telefonía inalámbrica, Internet, mensajería instantánea y las aplicaciones móviles (Apps), que posibilitan la comunicación y colaboración interpersonal (Distéfano, Mongelo, O'conor \& Lamas, 2015; Roussos \& Braun, 2018). Así, las TIC presentan características comunes fundamentales: interactividad, accesibilidad, alcance global y costos reducidos (Distéfano et al., 2015).

El incremento y uso de las tecnologías ha impactado de manera significativa el desarrollo e interacciones humanas a lo largo de las últimas décadas. $\mathrm{Al}$ respecto, Botella et al. (2007) aseguran que las nuevas tecnologías han ganado importancia y afirman que estos avances han mejorado nuestra calidad de vida, y se han introducido en diversos campos científicos en todos los ámbitos de la salud en general y en particular en la salud mental. Con ello, se ha potencializado la innovación para la evaluación, prevención e intervención psicológica, así como la formación de profesionales en esta área.

El término telesalud define el uso de las TIC para acceder a consulta, evaluación, intervención, supervisión, formación a distancia; se incluye términos como: telemedicina, telepsiquiatría y telepsicología (Lamas \& Distéfano, 2015; Peñate, 2012). Con la inclusión de las TIC se busca que la terapia llegue a todos aquellos que la necesiten para así reducir los malestares emocionales sin exceder el costo y con la misma eficacia (Botella et al., 2015). Asimismo, Peñate, Roca y Del Pino (2014) menciona que recursos como el e-mail, llamadas telefónicas, chats, videoconferencias y mensajes de texto han sido utilizados para proporcionar apoyo psicológico, consejería, seguimiento y/o tratamiento.

En este sentido, la teleterapia o telepsicoterapia consiste en llevar a cabo una intervención psicológica a distancia (González, Torres, Barrio \& Olmedo, 2017). Existe evidencia documentada de la eficacia y efectividad de dichos tratamientos ante determinados problemas psicológicos (Andersson, 2016; Botella et al., 2007); entre sus ventajas se encuentra que la movilidad es reducida, esto es de gran ayuda cuando el usuario presenta síntomas de alguna enfermedad o trastorno que lo lleve a permanecer en casa, hay mayor flexibilidad en cuanto al acceso y tiempo, y el costo de este tipo de terapia es más bajo (Peńate, Roca $\&$ Del Pino, 2014). Existe una 
distinción entre 3 tipos de teleterapia: la terapia a distancia que se lleva a cabo vía videoconferencia y teléfono; la terapia web que hace uso de programas autoaplicados sin apoyo de un terapeuta en tiempo real y, la terapia virtual que utiliza simulación de ambientes con o sin terapeuta de apoyo (González, Torres, Barrio \& Olmedo, 2017).

Así, la incorporación de Internet en los tratamientos psicológicos surge como una innovación en la psicoterapia asistida por computadoras que, por lo general, son autoadministrables, distribuidos vía plataformas digitales, aplicaciones web o móviles, accesibles en todo momento y desde cualquier lugar (Lamas \& Distéfano, 2015).

Los programas online y las aplicaciones móviles para la promoción de la salud mental requieren diseñarse específicamente para la población a la que estará dirigida La adolescencia es una etapa crítica en el desarrollo humano donde comienzan la mayoría de los trastornos mentales y frecuentemente permanecen sin tratamiento debido a la falta de búsqueda de ayuda y alfabetización para la salud emocional (de la Rosa, 2020). De este modo, el uso de tecnologías para la promoción, prevención e intervención en salud mental puede ser prometedora para la población más joven (Seko, Kidd, Wiljer \& McKenzie, 2014) debido a la familiaridad con dichas tecnologías que podría favorecer la motivación para su uso y potenciar la adherencia a las intervenciones (Baños et al., 2014).

Las aplicaciones de la psicoterapia vía internet para adolescentes son diversas y pueden brindarse con diferentes grados de participación o asistencia de un terapeuta. Lamas y Distéfano (2015) destacan que por lo general estas aplicaciones cuentan con tres componentes principales: 1) evaluación; 2) intervención, generalmente basada en terapia cognitivo-conductual (psicoeducación, reestructuración cognitiva, exposición y resolución de problemas); y 3) estrategias para la prevención de recaídas. La participación del usuario es esencial para que el programa funcione, $y$ se ha visto mayor eficacia y satisfacción en aquellos usuarios que se involucran lo suficiente. Actualmente la evidencia ha informado que los programas de prevención más eficaces son aquellos que integran técnicas interactivas y colaborativas (Rasal, 2014).

\section{Eficacia de los tratamientos vía internet}

Debido al gran crecimiento, uso e innovación de las nuevas tecnologías, en 1993 la división de Psicología Clínica de la Asociación Americana de Psicología (APA) creó un grupo de expertos para regular la psicoterapia mediada por las tecnologías, en 1995 elaboró una guía en la que estableció una distinción entre la eficacia de una intervención y su efectividad o utilidad clínica, que promovió el desarrollo y la difusión de tratamientos empíricamente validados con técnicas de tratamiento psicológico con respaldo empírico, sobresaliendo el uso de las técnicas cognitivo-conductuales (Botella et al., 2009). Para medir la eficacia se recomienda analizar con rigor la evidencia científica disponible de cualquier tratamiento y, para medir la efectividad sugiere indagar la generalización de los resultados de la intervención al contexto particular y cultural de la población meta (Botella et al., 2009).

La eficacia es el potencial que tiene un tratamiento para producir los cambios esperados, superiores a los resultados de no intervención o intervenciones estándar disponibles, además, los cambios se deben mantener a lo largo del tiempo. Así, permite conocer el grado de alcance de los propósitos establecidos en una intervención (Bados, García y Fuste, 2002; Mokate, 2001).

Sin duda, la eficacia de una intervención psicológica mediada por tecnología plantea una serie de desafíos: existe escasa formación para el entrenamiento de psicólogos clínicos y la apropiación de la tecnología como herramienta para las intervenciones; el riesgo de faltas éticas, la confidencialidad de datos sensibles se ve amenazada tanto para el terapeuta como para el usuario, malentendidos vinculados a fallos en la comunicación escrita asincrónica; abandono después de las primeras interacciones; identificación y gestión oportuna de crisis y riesgos de daños (Donker et al., 2013; Roussos \& Braun, 2018), así como el conseguir una tecnología adecuada y pertinente para brindar el apoyo psicológico oportuno a la población diana.

Dado que las investigaciones son incipientes en el contexto Latinoamericano y es relativamente escasa la literatura científica, el objetivo del presente estudio es realizar una revisión de las intervenciones eficaces vía internet para la salud emocional 
en los adolescentes y presentar una propuesta de intervención breve basada en técnicas de psicología positiva para potenciar la resiliencia ante la pandemia por COVID-19.

\section{MÉTODO}

Se realizó una búsqueda de artículos publicados sobre intervenciones eficaces vía internet para la salud emocional en los adolescentes entre el año 2010 al 2020 en las bases de datos PsycINFO, Scielo, PubMed y Google Scholar. La segunda etapa consistió en la revisión de los estudios para determinar cuáles cumplían con los criterios de inclusión: participantes entre 10 a 19 años; que el objetivo fueran intervenciones eficaces vía internet para la salud emocional en adolescentes y entregado a través de Internet o utilizando computadora o teléfono móvil. Se excluyeron los artículos que no reportaron resultados empíricos.

\section{Revisión de intervenciones eficaces vía internet para la salud emocional en los adolescentes}

Se encontraron 13 estudios científicos que cumplieron con los criterios de inclusión, cuyas características se presentan a continuación. La información detallada de los hallazgos de los estudios se presenta en la Tabla 1.

\section{Programas autoaplicados vía internet}

Stressbuster. Es un programa autoadministrado basado en la terapia cognitivo conductual que se compone de 8 sesiones contenidas en formato de CD-ROM, de 45 minutos cada una. Las sesiones son interactivas y multimedia con apoyo de videograbaciones, animaciones y gráficos que no requieren la participación de un terapeuta. Los videos son breves con un guión desarrollado ad hoc a la población adolescente. Durante la narración se incorporan conceptos claves que permiten ofrecer información sobre el sentir de un adolescente con sintomatología depresiva (Abeles et al., 2009).

Think, Feel, Do. Es un programa que se compone por 6 sesiones de 30 a 45 minutos de duración mediante formato de CD-ROM. Requiere el apoyo de un profesional como facilitador con entrenamiento en Terapia Cognitivo Conductual. Aborda componentes como: identificación de emociones, reestructuración cognitiva, regulación emocional y resolución de problemas. Los participantes interactúan con el sistema a través de la realización de pruebas y ejercicios que favorecen el aprendizaje de las estrategias de cambio conductual (Stallard, Richardson, Velleman, \& Attwood, 2011).

Yo Pienso, Siento, Actúo (YPSA). Programa semanal de 11 sesiones de 90 minutos cada una. Integra técnicas la Terapia Cognitivo Conductual, tales como, identificación de pensamientos negativos automáticos, regulación emocional y resolución de problemas. Está contenido en formato CD-ROM multimedia incluye cortos de películas, contenidos humorísticos de televisión y videos creados exprofeso para el programa (Martínez et al., 2014).

Bite Back. Es un programa autoaplicado que consiste en una variedad de actividades interactivas en línea durante 6 semanas que permite alentar la implementación de técnicas de psicología positiva en la vida diaria de los adolescentes. Propone estrategias como la gratitud, meditaciones de atención plena, describir historias personales y un ejercicio de atención plena. Las sesiones incluyen información sobre 9 dominios de la psicología positiva y sus formas de aplicación en la vida cotidiana, como: gratitud, optimismo, sentido de Fluir, significado, esperanza, atención plena, fortalezas de carácter, estilo de vida saludable y relaciones positivas. Los participantes pueden interactuar dentro del sitio web con un perfil anónimo, permitiendo así la participación sin temor al juicio o estigma. En todo momento se fomenta el respeto (Burckhardt et al., 2015).

CATCH-IT. Es un programa con 14 módulos para adolescentes y 4 módulos para padres que se brindan a través de Internet. Está basado en la Terapia Cognitivo Conductual con componentes relacionados a la activación conductual, relaciones interpersonales y resiliencia comunitaria. Se enseña a los adolescentes sobre la evitación, rumiación, estilo de comunicación indirecto para reducir pensamientos; así mismo, enseńa que el llevar una agenda de actividades y ampliar sus redes sociales de apoyo, entre otras estrategias, contrarrestan los 
pensamientos negativos y aumentan las estrategias de afrontamiento positivas ante la depresión. Se recomienda realizar 2 módulos por semana con duración entre 15 a 20 minutos. Incluye un manual para el padre/madre/tutor del adolescente con el propósito de brindar información sobre el vínculo entre las manifestaciones de depresión y la construcción de un perfil resiliente para afrontar los problemas de la vida en el ámbito personal y familiar. La aplicación del programa se precede una Entrevista Motivacional (EM) que es realizada por médicos entrenados en atención primaria con una duración entre 5 a 15 minutos, en donde se aborda el motivo de consulta personal, se informa cómo acceder a Internet para continuar la intervención; se realiza una visita de seguimiento y una entrevista entre 4 a 6 semanas. Los adolescentes participantes reciben 3 llamadas telefónicas de un trabajador social entrenado (Gladstone et al., 2015).

e - coach para la ansiedad y preocupación. Se brinda el programa a través de Internet con más de 6 sesiones de 30 a 40 minutos cada una, consta de 2 secciones principales: psicoeducación, que se presenta durante 2 sesiones con información sobre signos y síntomas de ansiedad generalizada, riesgo, factores, consecuencias, tratamientos médicos, psicológicos y de estilo de vida disponibles. Y, la segunda, estrategias para la reducción de la ansiedad que se brindan a partir de la tercera y cuarta sección; se presentan técnicas de la TCC centrándose en aspectos cognitivos de la preocupación y cómo abordarlos. Además, se entrena en técnicas de relajación a partir de la quinta sesión. Finalmente, se muestran los beneficios de ser físicamente activos y estrategias para aumentar o mantener su nivel actual de actividad física basado en la teoría del cambio (Calear et al., 2016).

Academia Espacial. Es un programa con énfasis en el desarrollo de la regulación emocional y la resiliencia. Existen dos versiones, una para niños $(6 \mathrm{a}$ 12 años) y otra para adolescentes (12 años o más). El tratamiento consta de 5 módulos, mediante la simulación de una academia espacial en la cual el usuario, a lo largo de diferentes misiones, avanza para ganar mayores rangos. El programa se ofrece en dos modalidades: software llamado MARTE que permite de manera presencial brindar apoyo psicoeducativoa a los adolescentes durante su estancia en el hospital; y a través de una Terapia Emocional Online (TEO) que refuerza los aprendizajes y potencia la práctica diaria de las estrategias enseñadas en MARTE (Flujas et al., 2017).

TOP. Programa de telesalud de mayor alcance. Es un programa de tratamiento basado en evidencia usado para tratar síntomas de estrés postraumático en niños y adolescentes de 3 a 18 años. Se administra entre 12-24 sesiones semanales e incluye: psicoeducación, estrategias de relajación, habilidades para la regulación emocional, habilidades de procesamiento cognitivo, procesamiento del trauma a partir de narrativas, exposición in vivo de los recuerdos traumáticos y, sesiones conjuntas entre niños y padres para la mejora de la percepción de seguridad y visión positiva del futuro. Además del material multimedia, ejercicios y juegos digitales, se realiza una videoconferencia semanal de $90 \mathrm{mi}$ nutos entre el terapeuta, el nińo o adolescente y el padre/madre/tutor (Stewart et al., 2020).

\section{Aplicaciones para teléfono móvil}

Diario del estado ánimo. Consiste en una aplicación JavaME que el participante descarga a su teléfono; está dirigida a adolescentes entre 10 a 17 años que acudieron a consulta a clínicas públicas de salud mental. A través de la aplicación se puede registrar el estado de ánimo y el nivel de entusiasmo en una escala de 1 a 10 , las horas de sueño en una escala de 1-18 horas, y también permite el registro de un diario de texto en formato libre, paso a paso. El programa en línea permite visualizar los estados de ánimo registrados y configurar recordatorios SMS personales. Los terapeutas monitorean continuamente las entradas de los participantes con un protocolo de seguimiento y asistencia (Mathew \& Doherty, 2011).

Seguimiento móvil de las experiencias de los jóvenes. Este programa fue escrito para su uso con múltiples modelos de teléfonos móviles. Monitorea el estado de ánimo, el estrés, las estrategias de afrontamiento y las actividades diarias de un joven varias veces al día, así como su alimentación, horas de sueño, patrones de ejercicio y consumo de alcohol y drogas una vez al día. Además, los participantes podrían notificar, a través de activar un botón de alerta, si están en riesgo de autolesio- 
narse o suicidarse y se derivan automáticamente a un psicólogo/consejero telefónico de guardia. El psicólogo contacta vía telefónica al joven y evalúa el riesgo de autolesión y alerta al equipo de asistencia comunitaria local del participante, si fuera necesario. Los datos recopilados por el programa se alojan en un sitio web seguro. Los datos también son cifrados y almacenados dentro de los teléfonos. Se han realizado diferentes estudios basados en el programa con éxito (Kauer et al., 2012; Reid et al., 2011; Silk et al., 2011).

Tratamiento móvil breve. El programa involucra una evaluación inicial cara a cara con los participantes. Consta de dos apartados. El primero incluye entrenamiento en: a) meditación (1 hora en total), respiración lenta, identificación de emociones, conductas y pensamientos disruptivos; b) resolución de problemas (30 a $60 \mathrm{~min}$ ); c) intervención breve para aumentar el apoyo social (30 a $60 \mathrm{~min}$ ); d) intervención breve para reducir el consumo de alcohol y otras drogas (30 a $60 \mathrm{~min}$ ). La segunda fase consiste en un seguimiento a distancia (móvil / web) comprendida por: a) 10 llamadas telefónicas de seguimiento después de haber finalizado la intervención, cuyo propósito es evaluar la tendencia suicida y el estado de ánimo, además, brindan una breve intervención centrada en la resolución de problemas, proporcionar orientación con respecto a la mejora del apoyo social, y alentar en el uso del mensajero o la línea de ayuda para obtener apoyo individual en caso de crisis (Marasinghe, Edirippulige, Kavanagh, Smith \& Jiffry, 2012).
Intervención preventiva para la depresión en adolescentes vía teléfono móvil (MEMO). Brindado por telefonía móvil basado en técnicas de terapia cognitivo conductual y aborda problemas comunes que enfrentan los adolescentes. Los adolescentes reciben 2 mensajes por día durante 9 semanas (fuera del horario escolar), seguido del envío de mensajes mensuales y acceso a un sitio web móvil de contenidos específicos sobre resolución de problemas, manejo de emociones y prevención de conductas de riesgo (Whittaker et al., 2012).

Tratamiento para la ansiedad infantil vía teléfonos inteligentes (Smart CAT). La aplicación para Android contempla cinco componentes principales: 1) notificación: avisa al participante cuando debe realizar ciertas tareas y proporciona estrategias psicoeducativas; 2) entrenamiento en habilidades para manejo de emociones, pensamientos negativos automáticos, estrategias afrontamiento, resolución de problemas, etc.; también el sistema envía alertas cuando los participantes experimentan ansiedad aguda; 3) banco de recompensas: los participantes pueden obtener premios por completar los módulos de entrenamiento a través de un sistema de puntos; 4) biblioteca de medios: incluye documentos, fotografías, videos y archivos de audio para ayudar a recordar y practicar las habilidades aprendidas en el tratamiento, y 5) mensajes seguros: para apoyar la interacción terapeuta-paciente dentro del portal del sistema (Pramana, Parmanto, Kendall \& Silk, 2014).

\section{Tabla 1}

Características de estudios basados en evidencia de intervenciones para la salud de adolescentes vía internet y aplicaciones móviles

\begin{tabular}{|c|c|c|c|c|c|}
\hline \multicolumn{6}{|c|}{ Programas autoaplicados vía Internet } \\
\hline $\begin{array}{c}\text { Autor, año y } \\
\text { país }\end{array}$ & Programa & Objetivo & Muestra & Diseño & Resultados \\
\hline $\begin{array}{l}\text { Abeles et al. } \\
\text { (2009). } \\
\text { Reino } \\
\text { Unido. }\end{array}$ & $\begin{array}{l}\text { Stressbus- } \\
\text { ters }\end{array}$ & $\begin{array}{l}\text { Evaluar preliminarmente el } \\
\text { tratamiento computarizado } \\
\text { de depresión en adolescen- } \\
\text { tes. }\end{array}$ & $\begin{array}{l}\mathrm{N}=23 \text { de } 12 \text { a } 16 \\
\text { años con diagnós- } \\
\text { tico de depresión } \\
\text { leve o moderada. }\end{array}$ & $\begin{array}{l}\text { Comparación } \\
\text { pre y post sin } \\
\text { grupo control. }\end{array}$ & $\begin{array}{l}\text { Al terminar } 78 \% \text { sin depre- } \\
\text { sión utilizando K-SADS. A } 3 \\
\text { meses } 93 \% \text { sin depresión. }\end{array}$ \\
\hline $\begin{array}{l}\text { Stallard et } \\
\text { al. (2011). } \\
\text { Reino } \\
\text { Unido. }\end{array}$ & $\begin{array}{l}\text { Think, Feel, } \\
\text { Do }\end{array}$ & $\begin{array}{l}\text { Describir el desarrollo de } \\
\text { una intervención cognitivo } \\
\text { conductual computariza- } \\
\text { da (Think, Feel, Do) para } \\
\text { jóvenes. }\end{array}$ & $\begin{array}{l}\mathrm{N}=20 \text { de } 11 \text { a } 16 \\
\text { años con trastor- } \\
\text { no de ansiedad o } \\
\text { depresión leve } 0 \\
\text { moderada. }\end{array}$ & $\begin{array}{l}\text { Ensayo } \\
\text { randomizado } \\
\text { controlado. }\end{array}$ & $\begin{array}{l}\text { Reducción significativa de } \\
\text { depresión contra el grupo } \\
\text { control en la escala AWS. } \\
\text { Satisfacción de moderada } \\
\text { a alta. }\end{array}$ \\
\hline
\end{tabular}




\begin{tabular}{|c|c|c|c|c|c|}
\hline \multicolumn{6}{|c|}{ Programas autoaplicados vía Internet } \\
\hline $\begin{array}{l}\text { Autor, año y } \\
\text { país }\end{array}$ & Programa & Objetivo & Muestra & Diseño & Resultados \\
\hline $\begin{array}{l}\text { Martínez et } \\
\text { al. (2014). } \\
\text { Chile. }\end{array}$ & $\begin{array}{l}\text { Yo Pienso, } \\
\text { Siento, } \\
\text { Actúo (YP- } \\
\text { SA-M). }\end{array}$ & $\begin{array}{l}\text { Comparar la eficacia de la } \\
\text { terapia cognitiva conductual } \\
\text { cara a cara tradicional con } \\
\text { un programa informático } \\
\text { versus la atención habitual } \\
\text { para tratar la depresión en } \\
\text { adolescentes en clínicas } \\
\text { de atención primaria en } \\
\text { Santiago, Chile. }\end{array}$ & $\begin{array}{l}\mathrm{N}=277 \text { estu- } \\
\text { diantes de } 90 \\
\text { grado, con edad } \\
\text { promedio de } 14.5 \\
\text { años. }\end{array}$ & $\begin{array}{l}\text { Ensayo } \\
\text { randomizado } \\
\text { controlado de } \\
\text { cuatro grupos } \\
\text { focales. }\end{array}$ & $\begin{array}{l}\text { Sin diferencia de la reduc- } \\
\text { ción de sintomatología } \\
\text { depresiva entre ambos gru- } \\
\text { pos BDI-Il a } 3 \text { y } 6 \text { meses, } \\
\text { la intervención fue factor } \\
\text { protector para depresión a } \\
\text { los } 6 \text { meses. Satisfacción } \\
\text { alta con el taller en evalua- } \\
\text { ción cualitativa. }\end{array}$ \\
\hline $\begin{array}{l}\text { Burckhardt } \\
\text { et al. (2015). } \\
\text { Australia. }\end{array}$ & Bite Back & $\begin{array}{l}\text { Alentar a los participantes a } \\
\text { trabajar a su máximo poten- } \\
\text { cial, participar activamente } \\
\text { en todos los ámbitos de } \\
\text { sus vidas y desarrollar la } \\
\text { resiliencia. }\end{array}$ & $\begin{array}{l}\mathrm{N}=338 \text { jóvenes } \\
\text { estudiantes de } \\
\text { entre } 12 \text { a } 18 \\
\text { años. }\end{array}$ & $\begin{array}{l}\text { Ensayo } \\
\text { controlado } \\
\text { aleatorizado, } \\
\text { comparación } \\
\text { pre y post test } \\
\text { con grupo } \\
\text { control }\end{array}$ & $\begin{array}{l}\text { Para la escala DASS-21, } \\
\text { SLSS y SWEMWBS no } \\
\text { demostraron beneficios } \\
\text { significativos en compara- } \\
\text { ción con una intervención } \\
\text { de control, esto no refleja } \\
\text { la eficacia del programa, } \\
\text { sino un problema en su } \\
\text { aplicación en el ambiente } \\
\text { escolar. }\end{array}$ \\
\hline $\begin{array}{l}\text { Gladstone et } \\
\text { al. (2015). } \\
\text { Estados } \\
\text { Unidos. }\end{array}$ & CATCH-IT & $\begin{array}{l}\text { Prevenir la depresión en } \\
\text { adolescentes y jóvenes } \\
\text { entre } 14 \text { y } 21 \text { años con } \\
\text { sintomatología subclínica. }\end{array}$ & $\begin{array}{l}\mathrm{N}=234 \text { de } 13 \text { a } \\
18 \text { años con pun- } \\
\text { tajes elevados de } \\
\text { estado de ánimo } \\
\text { deprimido. } \mathrm{N}= \\
186 \text { padres. }\end{array}$ & $\begin{array}{l}\text { Ensayo clínico } \\
\text { aleatorizado }\end{array}$ & $\begin{array}{l}\text { Significativa reducción pos } \\
\text { tratamiento de los sínto- } \\
\text { mas depresivos utilizando } \\
\text { CES-D }\end{array}$ \\
\hline $\begin{array}{l}\text { Calear, et al. } \\
\text { (2016). } \\
\text { Australia. }\end{array}$ & $\begin{array}{l}\text { E-Coach. } \\
\text { Ansiedad y } \\
\text { preocupa- } \\
\text { ción }\end{array}$ & $\begin{array}{l}\text { Evaluar la efectividad de un } \\
\text { programa de prevención de } \\
\text { ansiedad autodirigido en } \\
\text { línea. }\end{array}$ & $\begin{array}{l}\mathrm{N}=1,767 \\
\text { estudiantes de } \\
\text { secundaria de } 12 \\
\text { a } 18 \text { años. }\end{array}$ & $\begin{array}{l}\text { Ensayo } \\
\text { controlado } \\
\text { aleatorio } \\
\text { estratificado } \\
\text { por conglome- } \\
\text { rados de tres } \\
\text { brazos con } \\
30 \text { escuelas } \\
\text { australianas. }\end{array}$ & $\begin{array}{l}\text { No tuvo un efecto positivo } \\
\text { significativo en la salud } \\
\text { mental o el bienestar de los } \\
\text { participantes. La incor- } \\
\text { poración de un oficial de } \\
\text { educación en salud mental } \\
\text { para apoyar a los maestros } \\
\text { de clase en la entrega del } \\
\text { programa tampoco tuvo } \\
\text { efecto en los resultados de } \\
\text { la intervención. }\end{array}$ \\
\hline $\begin{array}{l}\text { Flujas et al. } \\
\text { (2017). } \\
\text { España. }\end{array}$ & $\begin{array}{l}\text { Academia } \\
\text { Espacial }\end{array}$ & $\begin{array}{l}\text { Programa de tratamiento } \\
\text { basado en realidad virtual en } \\
\text { línea para la disminución del } \\
\text { malestar emocional en niños } \\
\text { y adolescentes hospitaliza- } \\
\text { dos }\end{array}$ & $\begin{array}{l}\mathrm{N}=6 \text { pacientes } \\
\text { con enfermeda- } \\
\text { des de estancia } \\
\text { hospitalaria larga, } \\
\text { edad entre los } 9 \text { y } \\
16 \text { años. }\end{array}$ & Estudio Piloto & $\begin{array}{l}\text { El } 50 \% \text { de la muestra } \\
\text { redujo su puntuación en } \\
\text { afecto negativo, evitación } \\
\text { y aceptación; el } 50 \% \text { de } \\
\text { los pacientes aumentan su } \\
\text { resiliencia. }\end{array}$ \\
\hline $\begin{array}{l}\text { Stewart et } \\
\text { al. (2020). } \\
\text { Estados } \\
\text { Unidos. }\end{array}$ & $\begin{array}{l}\text { TOP. } \\
\text { Programa } \\
\text { de telesalud } \\
\text { de mayor } \\
\text { alcance }\end{array}$ & $\begin{array}{l}\text { Examinar la factibilidad y la } \\
\text { efectividad potencial de la } \\
\text { terapia cognitivo-conductual } \\
\text { centrada en el trauma admi- } \\
\text { nistrada por telepsicoterapia } \\
\text { en ubicaciones comunitarias } \\
\text { de escuelas u hogares. }\end{array}$ & $\begin{array}{l}\mathrm{N}=70 \text { niños y } \\
\text { adolescentes } \\
\text { de } 7 \text { a } 18 \text { años } \\
\text { remitidos de un } \\
\text { centro médico en } \\
\text { Carolina del Sur. }\end{array}$ & Estudio piloto & $\begin{array}{l}54 \text { participantes }(77.1 \%) \\
\text { evidenciaron cambios sus- } \\
\text { tanciales en los síntomas } \\
\text { postraumáticos autoinfor- } \\
\text { mados en el UCLA-TEPT- } \\
\text { IR }\end{array}$ \\
\hline
\end{tabular}




\begin{tabular}{|c|c|c|c|c|c|}
\hline \multicolumn{6}{|c|}{ Aplicaciones para teléfono móvil } \\
\hline $\begin{array}{c}\text { Autor, año y } \\
\text { país }\end{array}$ & Programa & Objetivo & Muestra & Diseño & Resultados \\
\hline $\begin{array}{l}\text { Matthews } \\
\text { y Doherty } \\
\text { (2011). } \\
\text { Irlanda. }\end{array}$ & $\begin{array}{l}\text { Stressbus- } \\
\text { ters. Diario } \\
\text { del estado } \\
\text { ánimo. }\end{array}$ & $\begin{array}{l}\text { Evaluar preliminarmente el } \\
\text { tratamiento computarizado } \\
\text { de depresión en adoles- } \\
\text { centes. }\end{array}$ & $\begin{array}{l}\mathrm{N}=23 \text { de } 12 \text { a } 16 \\
\text { años con diagnós- } \\
\text { tico de depresión } \\
\text { leve o moderada. }\end{array}$ & $\begin{array}{l}\text { Comparación } \\
\text { pre y post sin } \\
\text { grupo control. }\end{array}$ & $\begin{array}{l}\text { Al terminar } 78 \% \text { sin depre- } \\
\text { sión utilizando K-SADS. A } 3 \\
\text { meses } 93 \% \text { sin depresión. }\end{array}$ \\
\hline $\begin{array}{l}\text { Reid et } \\
\text { al. (2011). } \\
\text { Australia. }\end{array}$ & $\begin{array}{l}\text { Seguimien- } \\
\text { to móvil de } \\
\text { las expe- } \\
\text { riencias de } \\
\text { los jóvenes. }\end{array}$ & $\begin{array}{l}\text { Realizar un ensayo de } \\
\text { eficacia para evaluar la } \\
\text { utilidad del programa de } \\
\text { tipo móvil en el entorno } \\
\text { de atención primaria y } \\
\text { examinar los resultados de } \\
\text { salud mental. }\end{array}$ & $\begin{array}{l}\mathrm{N}=114 \text { pacien- } \\
\text { tes de } 14 \text { a } 24 \\
\text { años, el grupo de } \\
\text { comparación usó } \\
\text { una versión breve } \\
\text { de la herramienta } \\
\text { de autocontrol. }\end{array}$ & $\begin{array}{l}\text { Ensayo } \\
\text { controlado, } \\
\text { multicéntrico, } \\
\text { multirregional, } \\
\text { estratifica- } \\
\text { do (según } \\
\text { la región), } \\
\text { ciego simple, } \\
\text { controlado por } \\
\text { atención con } \\
\text { balance (1:1) } \\
\text { aleatorización } \\
\text { individual } \\
\text { en grupos } \\
\text { paralelos. }\end{array}$ & $\begin{array}{l}\text { La intervención aumen- } \\
\text { tó significativamente la } \\
\text { autoconciencia emocional. } \\
\text { Efecto principal significativo } \\
\text { de mediano a grande para } \\
\text { el tiempo de depresión, } \\
\text { ansiedad o estrés. }\end{array}$ \\
\hline $\begin{array}{l}\text { Silk et al. } \\
(2011) . \\
\text { Estados } \\
\text { Unidos. }\end{array}$ & $\begin{array}{l}\text { Seguimien- } \\
\text { to móvil de } \\
\text { las expe- } \\
\text { riencias de } \\
\text { los jóvenes. }\end{array}$ & $\begin{array}{l}\text { Investigar la eficacia del } \\
\text { uso del teléfono celular } \\
\text { para el monitoreo emocio- } \\
\text { nal diario de la población } \\
\text { clínica joven con depresión. }\end{array}$ & $\begin{array}{l}\mathrm{N}=47 \text { de } 7 \text { a } 17 \\
\text { años con tras- } \\
\text { torno depresivo } \\
\text { mayor (MDD) y } \\
32 \text { sin controles } \\
\text { de psicopatología } \\
(\mathrm{CON}) \text {. }\end{array}$ & $\begin{array}{l}\text { Estudio de } \\
\text { casos y con- } \\
\text { troles. }\end{array}$ & $\begin{array}{l}\text { Alta finalización (92\%). } \\
\text { El grupo de intervención } \\
\text { experimentó emociones } \\
\text { negativas más intensas } \\
\text { (tristeza, ira, nerviosismo, } \\
\text { afecto negativo global) que } \\
\text { el grupo de control. }\end{array}$ \\
\hline $\begin{array}{l}\text { Marasinghe } \\
\text { et al. (2012). } \\
\text { Australia. }\end{array}$ & $\begin{array}{l}\text { Tratamiento } \\
\text { móvil breve } \\
(\mathrm{BMT})\end{array}$ & $\begin{array}{l}\text { Examinar la eficacia de la } \\
\text { herramienta de autocontrol } \\
\text { basada en teléfonos móvi- } \\
\text { les en población joven con } \\
\text { síntomas depresivos. }\end{array}$ & $\begin{array}{l}\mathrm{N}=114 \text { de } 14 \text { a } \\
24 \text { años con pro- } \\
\text { blemas de salud } \\
\text { mental. }\end{array}$ & $\begin{array}{l}\text { Ensayo } \\
\text { Controlado } \\
\text { Aleatorio }\end{array}$ & $\begin{array}{l}\text { El autocontrol del estado } \\
\text { de ánimo aumentó signi- } \\
\text { ficativamente la autocon- } \\
\text { ciencia emocional (AE). Un } \\
\text { aumento de AE predijo una } \\
\text { disminución de los síntomas } \\
\text { depresivos. }\end{array}$ \\
\hline $\begin{array}{l}\text { Whittaker et } \\
\text { al. (2012). } \\
\text { Nueva } \\
\text { Zelanda. }\end{array}$ & $\begin{array}{l}\text { Intervención } \\
\text { preventiva } \\
\text { para la de- } \\
\text { presión en } \\
\text { adolescen- } \\
\text { tes vía telé- } \\
\text { fono móvil } \\
\text { (MEMO) }\end{array}$ & $\begin{array}{l}\text { Evaluar la eficacia de una } \\
\text { intervención basada en } \\
\text { TCC vía teléfono móvil } \\
\text { para usuarios con sinto- } \\
\text { matología depresiva en } \\
\text { comparación con un grupo } \\
\text { de control }\end{array}$ & $\begin{array}{l}\mathrm{N}=855 \text { estudian- } \\
\text { tes de } 13 \text { a } 17 \\
\text { años. }\end{array}$ & $\begin{array}{l}\text { Ensayo } \\
\text { Controlado } \\
\text { Aleatorio } \\
\text { doble ciego }\end{array}$ & $\begin{array}{l}\text { El } 90.7 \%(379 / 418) \text { del gru- } \\
\text { po de intervención favoreció } \\
\text { el programa, dicha interven- } \\
\text { ción los ayudó a ser más po- } \\
\text { sitivos }(279 / 418 ; 66.7 \%) \text { y a } \\
\text { los pensamientos negativos } \\
(210 / 418 ; 50.2 \%) \text {. }\end{array}$ \\
\hline $\begin{array}{l}\text { Pramana et } \\
\text { al. (2014). } \\
\text { Estados } \\
\text { Unidos. }\end{array}$ & $\begin{array}{l}\text { Tratamiento } \\
\text { para la } \\
\text { ansiedad } \\
\text { infantil vía } \\
\text { teléfonos } \\
\text { inteligentes } \\
\text { (Smart } \\
\text { CAT) }\end{array}$ & $\begin{array}{l}\text { Desarrollar una plataforma } \\
\text { de salud móvil para apoyar } \\
\text { el tratamiento de TCC } \\
\text { dirigido por el médico para } \\
\text { mejorar la adquisición y } \\
\text { utilización de habilidades } \\
\text { de TCC, promover el } \\
\text { entrenamiento de habilida- } \\
\text { des in vivo y monitorear la } \\
\text { utilización de habilidades } \\
\text { de los pacientes. }\end{array}$ & $\begin{array}{l}\mathrm{N}=9 \text { participantes } \\
\text { de } 9 \text { a } 14 \text { años. }\end{array}$ & $\begin{array}{l}\text { Ensayo clínico } \\
\text { aleatorizado, } \\
\text { simple ciego } \\
\text { con un grupo } \\
\text { control en lista } \\
\text { de espera. }\end{array}$ & $\begin{array}{l}\text { El estudio piloto muestra } \\
\text { comentarios positivos de pa- } \\
\text { cientes y terapeutas sobre } \\
\text { la viabilidad y efectividad de } \\
\text { la intervención usando un } \\
\text { protocolo de comunicación } \\
\text { avanzado para apoyar Coa- } \\
\text { ching de habilidades en vivo } \\
\text { y monitoreo del tratamiento } \\
\text { de niños con ansiedad.. }\end{array}$ \\
\hline
\end{tabular}


Nota: K-SADS: Programa para trastornos afectivos y esquizofrenia para niños en edad escolar; AWS: Escala de bienestar adolescente; BDI-II: Inventario de depresión de Beck;CES-D: Escala de depresión del Centro de Estudios Epidemiológicos; DASS-21: escala de depresión, ansiedad y estrés versión corta; SLSS: Escala de Satisfacción de la Vida Estudiantil; SWEMWBS: Escala corta de Bienestar Mental de Warwick-Edimburgo; CED-S: Escala de depresión del Centro de Estudios Epidemiológicos; UCLA-PTSD-RI exposición al trauma y los síntomas de estrés postraumático correspondientes a los criterios del Manual Diagnóstico y Estadístico de los Trastornos Mentales en jóvenes entre las edades de 7 y 18; BSSI: Escala de Beck para ideación suicida; MOS encuesta de apoyo social; AUDIT: Prueba de identificación de trastornos por consumo de alcohol; DCPL: Lista de problemas de verificación de drogas; SDS: Escala de gravedad de la dependencia.

Propuesta de intervención breve basada en técnicas de psicología positiva para potenciar la resiliencia ante la pandemia COVID-19

A partir de la revisión de la eficacia de las intervenciones mediadas por tecnología dirigidas a adolescentes, destacamos la evidencia existente de los programas de intervención autoaplicados vía internet cuya característica principal es ser autoadministrables y funcionar sin apoyo de un terapeuta en tiempo real. Dichas intervenciones adquieren relevancia en el contexto actual debido a la contingencia que se presenta por la pandemia por COVID-19, dado que permite que la psicoterapia llegue a todos aquellos que la necesiten sin tener que salir de casa, proporciona una mayor flexibilidad en cuanto al acceso y tiempo del tratamiento, así como una disminución del costo sin disminuir la calidad del servicio psicológico (Botella et al., 2015; Lorenzo, Díaz \& Zaldívar, 2020; Peńate et al., 2014).

Este tipo de programas brindados a través de internet han sido utilizados para la prevención de la salud mental, y así, intervenir en la población vulnerable como son los adolescentes para desarrollar habilidades para la vida, lo cual impactará en la disminución de la presencia de psicopatología.

Por otro lado, la Psicología Positiva es un movimiento científico desarrollado por Martín Seligman en 1998, que propone complementar el campo de la psicología con un nuevo objetivo: "el de explorar lo que hace que la vida valga la pena y crear las condiciones habilitadoras de una vida digna de ser vivida” (Seligman, 2016, p. 26). Para Park, Peterson y Sun (2013) es un enfoque que estudia el funcionamiento óptimo de las personas para dar lo mejor de sí mismas y puede dividirse en cuatro áreas relacionadas entre sí: (1) experiencias subjetivas positivas (felicidad/ bienestar, plenitud, fluir); (2) rasgos individuales positivos (fortalezas de carácter, talentos, intereses, valores); (3) relaciones interpersonales positivas (amistad, matrimonio, compañerismo) y (4) instituciones positivas (familias, escuelas, negocios, comunidades). Así, propone potenciar los aspectos saludables de las personas para prevenir la enfermedad, sin embargo, no niega los aspectos de enfermedad o de psicopatología que se pueden presentar después de un evento adverso.

De acuerdo con Tarragona (2013), los temas que se han investigado con este enfoque se encuentran: el bienestar subjetivo, el optimismo, la gratitud, la esperanza, la resiliencia, las emociones positivas, las fortalezas de carácter y los talentos, el sentido de vida y los valores, el humor, el bienestar en el trabajo, las parejas felices, la sabiduría y envejecer bien, entre otros temas.

Las intervenciones basadas en Psicología Positiva desarrollada por Martín Seligman en colaboración de otros académicos (Hérvas, 2009) han mostrado su mayor eficacia en la prevención y promoción de la salud en diversos problemas psicológicos con el objetivo de mejorar el bienestar y potenciar la resiliencia mediante la promoción de emociones positivas utilizando las fortalezas de carácter, el optimismo y la gratitud (Bohlmeijer, Bolier, Lamers \& Westerhof, 2017). Algunos programas probados se centran en emociones positivas orientadas al bienestar, terapia del bienestar, psicoterapia positiva para situaciones adversas, terapia centrada en el futuro, terapia de calidad de vida, psicoterapia positiva para la depresión, etc. (Arias, 2013). Asimismo, se están realizando intervenciones positivas en formato online como estrategia de salud pública mental, encontrando efectos significativos tanto en el bienestar emocional como en la depresión (Bohlmeijer, Bolier, Lamers \& Westerhof, 2017).

Debido a la eficacia y efectividad que han presentado los programas de intervención online autoaplicados para diversos problemas emocionales, 
se presenta una propuesta de intervención breve basada en técnicas de psicología positiva para potenciar la resiliencia para adolescentes que se encuentren en confinamiento durante la pandemia de COVID-19.

\section{Propuesta de Intervención}

La propuesta de intervención breve está basada en técnicas de psicología positiva para potenciar la resiliencia ante un desastre natural, dentro del cual se encuentra la pandemia por COVID-19, estresor de tipo biológico (Capacci \& Mangano, 2015). Consiste en un programa autoaplicado a través de internet que considera los siguientes 11 componentes terapéuticos (figura 1): Módulo 1. ¿Qué es un desastre natural?; Módulo 2. Conocimiento emocional; Módulo 3. Regulación emocional; Módulo 4. Motivación para el cambio; Módulo 5. Virtudes y fortalezas; Módulo 6. Aceptación y gratitud; Módulo 7. Optimismo y persistencia; Módulo 8. Resiliencia; Módulo 9. Crecimiento postraumático; Módulo 10. Habilidades conductuales y Módulo 11. Redes de Apoyo. Cabe señalar que los títulos de cada uno de los módulos fueron modificados para que fueran atractivos para los adolescentes, así mismo, contiene objetivos específicos de intervención, presentación de contenido a través de infografías y vídeos, ejercicios para el desarrollo de habilidades y al finalizar cada uno de los módulos el usuario ganará una recompensa. Finalmente, la secuencia del programa no permite que el usuario pueda avanzar de un módulo a otro sin haberlo cursado (ver tabla 2).

Adicional a los componentes terapéuticos, el programa tendrá un manual de uso que consiste en una guía práctica que le permitirá al usuario conocer: 1) los requisitos técnicos para utilizar el programa autoaplicado, 2) los requisitos previos para acceder al programa de intervención que corresponde al consentimiento informado de los padres o tutores así como el consentimiento personal, 3) responder la evaluación inicial y final del programa de intervención, 4) conocer el tiempo en que el usuario podrá elaborar cada una de las actividades, sin embargo, este puede adecuarse a las necesidades de los adolescentes.

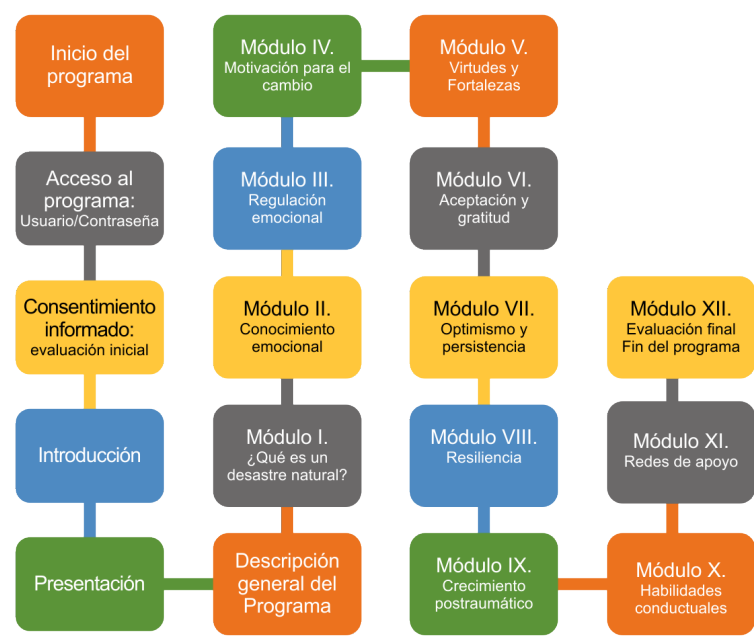

Figura 1. Diagrama de flujo y componentes del programa autoaplicado.

Fuente: Elaboración propia (2020)

Tabla 2

Programa de intervención breve basada en técnicas de psicología positiva

\section{Programas autoaplicados vía Internet}

\begin{tabular}{|c|c|c|}
\hline $\begin{array}{l}\text { Módulos de } \\
\text { intervención }\end{array}$ & $\begin{array}{l}\text { Objetivo } \\
\text { específico }\end{array}$ & Actividad \\
\hline $\begin{array}{l}\text { Módulo } \\
\text { I. Cono- } \\
\text { ciendo los } \\
\text { desastres } \\
\text { naturales. }\end{array}$ & $\begin{array}{l}\text { Conocer los desas- } \\
\text { tres naturales prin- } \\
\text { cipales en México } \\
\text { (sismos, terremotos, } \\
\text { huracanes). }\end{array}$ & $\begin{array}{l}\text { Realizar el ejercicio: } \\
\text { "Sopa de letras" } \\
\text { para identificar el } \\
\text { conocimiento sobre } \\
\text { el tema en desastres } \\
\text { naturales. }\end{array}$ \\
\hline $\begin{array}{l}\text { Módulo II. } \\
\text { ¿Qué pasa } \\
\text { con mis } \\
\text { emociones }\end{array}$ & $\begin{array}{l}\text { Identificar emocio- } \\
\text { nes, su función y ser } \\
\text { consciente de ellas. }\end{array}$ & $\begin{array}{l}\text { Registrar e identificar } \\
\text { las sensaciones, } \\
\text { emociones y conduc- } \\
\text { tas que se presentan } \\
\text { durante el día. }\end{array}$ \\
\hline $\begin{array}{l}\text { Módulo III. } \\
\text { ¿Cómo me } \\
\text { controlo? }\end{array}$ & $\begin{array}{l}\text { Conocer la regula- } \\
\text { ción emocional, así } \\
\text { como la capacidad } \\
\text { para controlar y } \\
\text { expresar emociones }\end{array}$ & $\begin{array}{l}\text { Reconocer y escribir } \\
\text { las emociones para } \\
\text { poderlas controlar y } \\
\text { expresar en diferen- } \\
\text { tes situaciones de la } \\
\text { vida cotidiana }\end{array}$ \\
\hline $\begin{array}{l}\text { Módulo IV. } \\
\text { Quiero } \\
\text { hacer un } \\
\text { cambio. }\end{array}$ & $\begin{array}{l}\text { Resaltar la importan- } \\
\text { cia de estar motiva- } \\
\text { do para conseguir un } \\
\text { cambio después de } \\
\text { la adversidad. }\end{array}$ & $\begin{array}{l}\text { Analizar y escribir los } \\
\text { pros y contras para } \\
\text { realizar un cambio } \\
\text { después de un even- } \\
\text { to adverso }\end{array}$ \\
\hline $\begin{array}{l}\text { Módulo V. } \\
\text { Hay más de } \\
\text { un camino. }\end{array}$ & $\begin{array}{l}\text { Identificar las emo- } \\
\text { ciones positivas, las } \\
\text { virtudes y fortalezas } \\
\text { psicológicas en } \\
\text { diversas situaciones } \\
\text { personales. }\end{array}$ & $\begin{array}{l}\text { Conocer las forta- } \\
\text { lezas de carácter y } \\
\text { reflexionar en qué } \\
\text { momentos de la vida } \\
\text { se han empleado. }\end{array}$ \\
\hline
\end{tabular}




\begin{tabular}{|c|c|c|}
\hline \multicolumn{3}{|c|}{ Programas autoaplicados vía Internet } \\
\hline $\begin{array}{l}\text { Módulos de } \\
\text { intervención }\end{array}$ & $\begin{array}{l}\text { Objetivo } \\
\text { específico }\end{array}$ & Actividad \\
\hline $\begin{array}{l}\text { Módulo VI. } \\
\text { Cadena de } \\
\text { regalos. }\end{array}$ & $\begin{array}{l}\text { Aceptar una } \\
\text { situación difícil y } \\
\text { agradecer por lo que } \\
\text { se tiene. }\end{array}$ & $\begin{array}{l}\text { Identificar situacio- } \\
\text { nes adversas que } \\
\text { se han aceptado y } \\
\text { reflexionar sobre } \\
\text { las estrategias para } \\
\text { afrontarlas, ponerlas } \\
\text { en práctica en otras } \\
\text { situaciones difíciles. }\end{array}$ \\
\hline $\begin{array}{l}\text { Módulo VII. } \\
\text { Sabías } \\
\text { que... el que } \\
\text { persevera } \\
\text { alcanza. }\end{array}$ & $\begin{array}{l}\text { Conocer los benefi- } \\
\text { cios de implementar } \\
\text { el optimismo y la } \\
\text { persistencia. }\end{array}$ & $\begin{array}{l}\text { Elegir un objetivo que } \\
\text { se desee cumplir, } \\
\text { escribir los factores } \\
\text { personales y contex- } \\
\text { tuales que impiden } \\
\text { cumplir con la meta. } \\
\text { Después diseñar una } \\
\text { estrategia que ayude } \\
\text { a neutralizarlos. } \\
\text { Pensar en tres cosas } \\
\text { que hayan salido bien } \\
\text { durante la semana } \\
\text { pasada. }\end{array}$ \\
\hline $\begin{array}{l}\text { Módulo VIII. } \\
\text { Trampas del } \\
\text { pensamien- } \\
\text { to. }\end{array}$ & $\begin{array}{l}\text { Identificar alterna- } \\
\text { tivas a las ideas } \\
\text { o pensamientos } \\
\text { negativos. }\end{array}$ & $\begin{array}{l}\text { Seleccionar una } \\
\text { situación problema, } \\
\text { identificar ideas o } \\
\text { creencias negativas y } \\
\text { reconocer la trampa } \\
\text { de pensamiento en } \\
\text { la que se ubican. } \\
\text { Después realizar una } \\
\text { interpretación alterna- } \\
\text { tiva de la creencia. }\end{array}$ \\
\hline $\begin{array}{l}\text { Módulo IX. } \\
\text { Floreciendo }\end{array}$ & $\begin{array}{l}\text { Aprender a identi- } \\
\text { ficar el problema y } \\
\text { cambiar su signifi- } \\
\text { cado. }\end{array}$ & $\begin{array}{l}\text { Pensar en los } \\
\text { resultados positivos } \\
\text { que han surgido de } \\
\text { situaciones adversas } \\
\text { e identificar aquellas } \\
\text { por las que se debe } \\
\text { sentir agradecido o } \\
\text { expresar gratitud. } \\
\text { Analizar las situacio- } \\
\text { nes difíciles que se } \\
\text { hayan enfrentado en } \\
\text { la vida y el aprendiza- } \\
\text { je o crecimiento que } \\
\text { se alcanzó. }\end{array}$ \\
\hline $\begin{array}{l}\text { Módulo X. } \\
\text { Durante el } \\
\text { movimiento. }\end{array}$ & $\begin{array}{l}\text { Conocer cómo } \\
\text { afrontar y solucionar } \\
\text { un problema en caso } \\
\text { de presentarse un } \\
\text { desastre natural. }\end{array}$ & $\begin{array}{l}\text { Elegir un problema } \\
\text { que pueda surgir de } \\
\text { un desastre natural, } \\
\text { analizar qué se nece- } \\
\text { sita para resolverlo y } \\
\text { dividirlo en unidades } \\
\text { pequeñas para } \\
\text { elaborar un plan de } \\
\text { cambio. }\end{array}$ \\
\hline
\end{tabular}

\begin{tabular}{lll}
\hline \multicolumn{3}{c}{ Programas autoaplicados vía Internet } \\
\hline $\begin{array}{l}\text { Módulos de } \\
\text { intervención }\end{array}$ & $\begin{array}{c}\text { Objetivo } \\
\text { específico }\end{array}$ & \multicolumn{1}{c}{ Actividad } \\
\hline $\begin{array}{l}\text { Módulo XI. } \\
\text { ¿Dónde }\end{array}$ & $\begin{array}{l}\text { Aprender a identifi- } \\
\text { car redes de apoyo }\end{array}$ & $\begin{array}{l}\text { Buscar a una perso- } \\
\text { na con la cual tener }\end{array}$ \\
busco & y de qué manera & una conversación y \\
apoyo? & formar relaciones & hablar sobre buenas \\
positivas. & $\begin{array}{l}\text { noticias, haciendo } \\
\text { contacto visual y } \\
\text { mostrando interés. }\end{array}$ \\
\hline
\end{tabular}

Nota: los nombres de los módulos fueron modificados para atraer la atención de los adolescentes.

En el módulo 12, se presentará un video con el cierre del programa de intervención, la evaluación final y la última recompensa. Una vez finalizado el programa el participante podrá revisar los módulos las veces que lo requiera.

\section{Conclusiones}

El presente trabajo tuvo por objetivo revisar la evidencia de intervenciones mediadas por internet y aplicaciones móviles que han mostrado eficacia en la intervención de salud emocional en adolescentes. Al respecto, se observó que las intervenciones vía internet han resultado ser novedosas e innovadoras; no obstante, no todos los países han alcanzado desarrollo e investigación robusta en el campo; en países Latinoamericanos, especialmente en México, se requiere generar un mayor grado de investigación y desarrollo tecnológico debido a que es incipiente.

En general, se destaca que las intervenciones vía internet obtienen resultados alentadores (Abeles et al., 2009; Gladstone et al., 2015; Kauer et al., 2012; Marasinghe et al., 2012; Martínez et al., 2014; Matthews \& Doherty, 2011; Pramana et al., 2014; Reid et al., 2011; Stallard et al., 2011; Whittaker et al., 2012); sin embargo, el acceso a estas intervenciones en ocasiones es limitado ya sea por la poca accesibilidad a los dispositivos y/o a una red de internet.

Entre los hallazgos, se encontró que las intervenciones guiadas por profesionales, ya sea con poco o mucho entrenamiento, son más eficaces que los tratamientos no guiados (Clear et al., 2016; Glads- 
tone et al., 2015; Stallard et al., 2011), y aunque todavía se encuentra en debate, lo que es cierto es que los tratamientos no guiados generan menos costes y pueden llegar a más personas mientras que los tratamientos guiados por un profesional de la salud parecen ser más eficaces pero tienen un grupo objetivo más reducido (Johansson \& Andersson, 2012).

Por otro lado, las tasas informadas de adherencia a la intervención fueron altas, en particular, al ofrecer el apoyo vía teléfono móvil (Kauer et al., 2012; Marasinghe et al., 2012; Matthews \& Doherty, 2011; Pramana et al., 2014; Reid et al., 2011; Silk et al., 2011; Whittaker et al., 2012) debido a la portabilidad, flexibilidad, y personalización. Asimismo, se mostraron tasas altas de la finalización del tratamiento, un monitoreo y seguimiento pertinente en las muestras clínicas de adolescentes, así como una mejor forma para divulgar y diseminar información pertinente y necesaria a los usuarios sin requerir que lean grandes cantidades de texto (Marasinghe et al., 2012; Matthews \& Doherty, 2011; Reid et al., 2011; Silk et al., 2011; Kauer et al., 2012). También se informó consistentemente que, aunque la intervención tradicional cara a cara tiene muchos beneficios y aceptación, es posible que los adolescentes sean menos propensos y tengan menos confianza a compartir experiencias privadas a otra persona que cuando usan un dispositivo electrónico (Marasinghe et al., 2012; Martínez et al., 2014; Stallard et al., 2011).

En este sentido, los resultados disponibles parecen prometedores y sugieren que la tecnología basada en internet se puede utilizar para administrar intervenciones preventivas para la salud emocional en los adolescentes y pueden mejorar significativamente la accesibilidad al tratamiento. No obstante, debido al tamaño de la muestra y al no uso de diseños controlados en algunos estudios, estos hallazgos deben considerarse con precaución a la espera de la replicación de sus resultados.

El desarrollo tecnológico ofrece a la práctica clínica diversidad, accesibilidad y mayor alcance, los tratamientos vía internet han demostrado ser eficaces $y$, también, se ha comprobado que son bien aceptados por los participantes, de esta manera, se han convertido en una propuesta conveniente y perti- nente. Derivado de esto, los contenidos se deben enfocar primordialmente en el desarrollo de programas breves, interactivos, presentados de forma novedosa que se adapten a las necesidades de los usuarios, ya que de esta manera se podrá potencializar el interés y la aceptación de los adolescentes.

En cuanto a los profesionales de la salud mental se vuelve necesaria la sensibilización en actitudes positivas al uso de tecnología en los tratamientos psicológicos, mayor difusión de su eficacia y entrenamiento adecuado para el análisis de factibilidad de la incorporación en su quehacer profesional particular.

En concreto, los programas auto-aplicados vía Internet, es una nueva modalidad para brindar apoyo psicológico que ha sido utilizada de manera creciente y que representa una alternativa y una herramienta poderosa, cuando: (a) no se tiene acceso a servicios de apoyo psicológico, (b) los jóvenes prefieren guardar el anonimato al principio del proceso de atención psicológica y (c) se tienen dificultades para identificar los beneficios del apoyo psicológico y de esta manera pueden tener el primer contacto con programas diseńados por profesionales de la salud. Es importante enfatizar que se requiere la expansión de su uso, diseminación e investigación robusta con el propósito de aumentar la confianza en las intervenciones mediadas por internet, y así garantizar resultados óptimos en la salud pública de la población adolescente.

Con lo anterior, es necesario el desarrollo de programas de intervención en psicología basados en la evidencia que favorezcan la disminución del impacto psicológico durante las diferentes fases de la epidemia por COVID-19 para el bienestar de la población, principalmente en la población vulnerable como los niños y los adolescentes (Lorenzo et al., 2020).

\section{Agradecimiento}

Investigación financiada por el proyecto PAPIIT <<TA300219>> Intervención online autoaplicada basada en técnicas de Psicología Positiva para la promoción de resiliencia ante desastres naturales. 


\section{REFERENCIAS BIBLIOGRÁFICAS}

Abeles, P., Verduyn, C., Robinson, A., Smith, P., Yule, W., \& Proudfoot, J. (2009). Computerized CBT for Adolescent Depression ("Stressbusters") and its Initial Evaluation Through an Extended Case Series. Behavioural and Cognitive Psychotherapy, 37(02), 151.

https://doi.org/10.1017/S1352465808005067

Andersson, G. (2016). Internet-Delivered Psychological Treatments. Annual Review of Clinical Psychology, 12, 157-179. https://doi.org/10.1146/annurev-clinpsy-021815-093006

Andrews, G., Cuijpers, P., Craske, M.G., McEvoy, P., \& Titov, N. (2010). Computer Therapy for the Anxiety and Depressive Disorders Is Effective, Acceptable and Practical Health Care: A Meta-Analysis. PLOS ONE, 5(10). https://doi.org/10.1371/journal.pone.0013196

Alvites-Huamaní, C. (2019). Adolescencia, ciberbullying y depresión, riesgos en un mundo globalizado. Revista Científica electrónica de Educación y Comunicación en la Sociedad del Conocimiento. 19 (1), 210-234. Recuperado de http://eticanet.org/revista/index.php/eticanet/article/ view/182/159

Alvites-Huamaní, C. (2020). Covid-19: Pandemia que impacta en los estados de Ánimo. CienciAmérica, 9 (2).

http://dx.doi.org/10.33210/ca.v9i2.327

Arias, W. (2013). Psicología clínica y psicoterapia: revisión epistemológica y aportes de la psicología positiva. Revista de Peruana de psicología y trabajo social, 2 (1), 137-154.

Bados, L., García, B., \& Fusté, E. (2002). Eficacia y utilidad clínica de la terapia psicológica. Revista Internacional de Psicología Clínica y de la Salud, 2(3), 477-502. Recuperado de https://psicopedia.org/wp-content/uploads/2014/11/Eficacia-terapia-psicologica.pdf

Baños, M., Etchemendy, E., Farfallini, L., García-Palacios, A., Quero, S., \& Botella, C. (2014). EARTH of Well-Being System: A pilot study of an Information and Communication Technology-based positive psychology intervention. The Journal of Positive Psychology, 9(6), 482-488. https:// doi.org/10.1080/17439760.2014.927906

Bohlmeijer, E.T , Bolier, L. Lamers S.M.A., y Westerhof, G. J (2017). Intervenciones clínicas positivas: ¿Por qué son importantes y cómo funcionan? Papeles del Psicólogo, 38(1), 34-41. https://doi.org/10.23923/pap.psicol2017.2819

Botella, C., Baños, R., García-Palacios, Quero, S., Guillén V., y José, M. (2007). La utilización de las nuevas tecnologías de la información y la comunicación en psicología. Revista sobre la sociedad del conocimiento, 4, 32-41. Recuperado de https://www.redalyc.org/articulo.oa?id=79000409

Botella, C., Mira, A., Herrero, R., García, A. y Baños, R. (2015). Un programa de intervención autoaplicado a través de internet para el tratamiento de la depresión: sonreír es divertido. Aloma, 33(2), 39-48.
Botella, C., Quero, S., Serrano, B., Baños, R., y García-Palacios, A. (2009). Avances en los tratamientos psicológicos: la utilización de las nuevas tecnologías de la información y la comunicación. Anuario de Psicología, 40 (2), 155-170. Recuperado de https://www.raco.cat $>$ index.php $>$ AnuarioPsicologia , article , download

Brooks, S. K., Webster, R. K., Smith, L. E., Woodland, L., Wessely, S., Greenberg, N., \& Rubin, G. J. (2020). The psychological impact of quarantine and how to reduce it: rapid review of the evidence. The Lancet, 395(10227), 912-920.

https://doi.org/10.1016/S0140-6736(20)30460-8

Burckhardt, R., Manicavasagar, V., Batterham, P., Miller, M., Talbot, E. y Lum, A. (2015). Un programa de psicología positiva para adolescentes basado en la web en las escuelas: ensayo controlado aleatorio. J Med Internet Res, 17 (7).

https://doi.org/10.2196/jmir.4329

Calear, A., Batterham, P., Poyser, C., Mackinnon, A., Griffiths, K., \& Christensen, H. (2016). Cluster randomised controlled trial of the e-couch Anxiety and Worry program in schools. Journal of Affective Disorders, 196, 210-217. https://doi.org/10.1016/j.jad.2016.02.049

Capacci, A. y Mangano, S. (2015) Las catástrofes naturales. Cuadernos de Geografía: Revista Colombiana de Geografía, 24(2), 35-51.

https://doi.org/10.15446/rcdg.v24n2.50206

Cárdenas, G., Botella, C., Quero, S., De la Rosa, A., y Bańos, R. (2014). Programa de Telepsicología para el Tratamiento de la Fobia a hablar en público en Población Mexicana. Revista Psicología Iberoamericana, 22(1).

Corona, H., \& Peralta, V. (2010). Prevención de conductas de riesgo. Rev. Med. Clin. Condes, 22(1), 68-75. Recuperado de https://www.clinicalascondes.cl/Dev_CLC/media/ Imagenes/PDF\%20reista\%20m\%C3\%A9dica/2011/1\%20 enero/8_Dra_Corona-10.pdf https://doi.org/10.1016/S0716-8640(11)70394-7

De la Rosa, N. (2020). Eficacia de intervenciones preventivas autoaplicadas vía internet para la salud emocional en los adolescentes. Una revisión (Tesis de licenciatura inédita). Universidad Nacional Autónoma de México, México.

Distéfano, M. J., Mongelo, M. C., O’Connor, J., y Lamas, M. C. (2015). Psicoterapia y tecnología: Implicancias y desafíos en la inserción de recursos innovadores en la práctica clínica Argentina. Revista Electrónica de Psicología Iztacala, 18 (4),1342-1362. Recuperado de http://www.revistas.unam. mx/index.php/repi/article/view/53433/47525

Donker, T., Petrie, K., Proudfoot, J., Clarke, J., Abedul, M., \& Christensen, H. (2013) Smartphones for smarter delivery of mental health programs: a systematic review. Revista de Investigación médica en Internet, 15 (11). https://doi.org/10.2196/jmir.2791

Ebert, D., Van Daele, T., Nordgreen, T., Karekla, M., Compare, A., Zarbo, C., Brugnera, A., Øverland, S., Trebbi, G., Jensen, K. L., Kaehlke, F. \& Baumeister, H. (2018). Internet- and Mobile-Based Psychological Interventions: Appli- 
cations, Efficacy, and Potential for Improving Mental Health. A Report of the EFPA E-Health Taskforce. European Psychologist, 23, 67-187. https://doi.org/10.1027/1016$9040 / \mathrm{a} 000318$

Flujas-Contreras, J., Ruiz-Castañeda, D., Botella, C. y Gómez, I. (2017). Un programa de bienestar emocional basado en Realidad Virtual y Terapia en línea para enfermedades crónicas en infancia y adolescencia: La Academia Espacial. Revista de Psicología Clínica con Niños y Adolescentes, 4 (3), 17-25. Recuperado de https://www.revistapcna.com/ sites/default/files/17-16.pdf

Gladstone, T., Marko-Holguin, M., Rothberg, P., Nidetz, J., Diehl, A., DeFrino, D., Harris, M., Ching, E., Eder, M., Cane, J., Bell, K., Beardslee, W., Brown, H., Griffiths, K., \& Van Voorhees, B. (2015). An internet-based adolescent depression preventive intervention: study protocol for a randomized control trial. Trials, 16(1).

https://doi.org/10.1186/s13063-015-0705-2

González-Peña, P., Torres, R., Del Barrio, V., y Olmedo, M. (2017). Uso de las nuevas tecnologías por parte de los psicólogos españoles y sus necesidades. Clínica y salud, 28, 81-91. https://doi.org/10.1016/j.clysa.2017.01.001

Herrero, R., Mira, A., Cormo, G., Etchemendy, E., Baños, R., García-Palacios, A., Ebert, D., Franke, M., Berger, T., Schaub, M., Görlich, Jacob, C. \& Botella, C. (2018, in press). An Internet based intervention for improving resilience and coping strategies in university students: Study protocol for a randomized controlled trial. Internet Interventions. https://doi.org/10.1016/j.invent.2018.03.005

Hervás, G. (2009). Psicología positiva: una introducción. Revista Interuniversitaria de Formación del Profesorado, 23 (3), 23-41. Recuperado de https://www.redalyc.org/articulo. oa?id=274/27419066003

Johansson, R., y Andersson, G. (2012). Internet-based psychological treatments for depression. Expert Review of Neu. rotherapeutics, 12(7), 861-870.

https://doi.org/10.1586/ern.12.63

Kauer, S., Reid, S., Crooke, A., Khor, A., Hearps, S., Jorm, A., Sanci, L., \& Patton, G. (2012). Self-monitoring Using Mobile Phones in the Early Stages of Adolescent Depression: Randomized Controlled Trial. J Med Internet Res, 14(3). https://doi.org/10.2196/jmir.1858

Lamas, M. C., \& Distéfano, M. J. (2015). Psicología y Tecnología en el nuevo siglo. En A. Martínez-Núñez, V. A., Godoy, P., Piñeda, M. A., Fantín, M. B., Cuello, M., Bower, L., De Andrea, N., González, E., Katzer, N. \& Morales, L. E. (Eds.). Avances y Desafíos para la Psicología, 411-421. Argentina: Nueva Editorial Universitaria.

Li, S., Wang, Y., Xue, J., Zhao, N., \& Zhu, T. (2020). The impact of COVID-19 epidemic declaration on psychological consequences: a study on active Weibo users. International journal of environmental research and public health, 17(6), 2032.

https://doi.org/10.3390/ijerph17062032
Lorenzo, R. A., Díaz, A. K., y Zaldívar, P. D. (2020). La psicología como ciencia en el afrontamiento a la COVID-19: apuntes generales. Anales de la Academia de Ciencias de Cuba, 10 (2). https://doi.org/10.37226/rcp.v4i2.4815

Marasinghe, R., Edirippulige, S., Kavanagh, D., Smith, A., \& Jiffry, M. (2012). Effect of mobile phone-based psychotherapy in suicide prevention: a randomized controlled trial in Sri Lanka. Journal of Telemedicine and Telecare, 18(3), 151-155.

https://doi.org/10.1258/jtt.2012.SFT107

Martínez, V., Martínez, P., Vöhringer, P., Araya, R., \& Rojas, G. (2014). Computer-assisted cognitive-behavioral therapy for adolescent depression in primary care clinics in Santiago, Chile (YPSA-M): study protocol for a randomized controlled trial. BMC Family Practice. https://doi.org/10.1186/17456215-15-309

Matthews, M., \& Doherty, G. (2011). In the mood: engaging teenagers in psychotherapy using mobile phones. https://doi.org/10.1145/1978942.1979379

Mokate, K. (2001). Eficacia, Eficiencia, equidad y sostenibilidad: ¿Qué queremos decir? Banco Interamericano de Desarrollo.

Park, N., Peterson, C., \& Sun, J. (2013). La psicología positiva: investigación y aplicaciones. Terapia psicológica, 31(1), 11-19. https://doi.org/10.4067/S071848082013000100002

Pasarelu, C., Andersson, G., Bergman, L., \& Dobrean, A. (2017). Internet-delivered transdiagnostic and tailored cognitive behavioral therapy for anxiety and depression: a systematic review and meta-analysis of randomized controlled trials. Cognitive Behaviour Therapy, 46(1), 1-28. https:// doi.org/10.1080/16506073.2016.1231219

Peñate, C., Roca, S., y Del Pino, S. (2014). Los nuevos desarrollos tecnológicos aplicados al tratamiento psicológico. Acta Colombiana de Psicología, 17 (2), 91-101.

https://doi.org/10.14718/ACP.2014.17.2.10

Peñate, W. (2012). About the effectiveness of telehealth procedures in psychological treatments. International Journal of Clinical and Health Psychology, 12 (3), 475-487.

Pramana, G., Parmanto, B., Kendall, P. C., \& Silk, J., S. (2014). The SmartCAT: An m-Health Platform for Ecological Momentary Intervention in Child Anxiety Treatment. Telemedicine and e-Health, 20(5), 419-427.

https://doi.org/10.1089/tmj.2013.0214

Rasal. (2014). Un programa autoaplicado para la prevención del consumo de drogas en población adolescente en el contexto escolar (tesis doctoral). Universidad de Valencia, España.

Reid, S., Kauer, S., Hearps, S., Crooke, A., Khor, A., Sanci, L., \& Patton, G. C. (2011). A mobile phone application for the assessment and management of youth mental health problems in primary care: a randomised controlled trial. BMC Práctica familiar, 12 (1). https://doi.org/10.1186/14712296-12-131 
Roussos, A., \& Braun, M. (2018). Psicoterapia y tecnologías de información y comunicación. Psicoterapia 2.0. Enciclopedia Argentina de la Salud Mental. 1. Recuperado de http://www.enciclopediasaludmental.org.ar/trabajo.php?id$\mathrm{t}=49 \& \mathrm{idt} \mathrm{t}=7$

Seko, Y., Kidd, S., Wiljer, D., \& McKenzie, K. (2014). Youth Mental Health Interventions via Mobile Phones: A Scoping Review. Ciber Psicología, comportamiento y redes sociales, 17(9), 591-602. https://doi.org/10.1089/cyber.2014.0078

Seligman, M. (2016). Florecer. La nueva psicología positiva y la búsqueda del bienestar. México-Océano exprés.

Silk, J., Forbes, E., Whalen, D., Jakubcak, J., Thompson, W., Ryan, N., Axelson, D., Birmaher, B., \& Dahl, R. (2011). Daily emotional dynamics in depressed youth: A cell phone ecological momentary assessment study. Journal of Experimental Child Psychology, 110(2), 241-257. https://doi.org/10.1016/j.jecp.2010.10.007

Stallard, P., Richardson, T., Velleman, S., \& Attwood, M. (2011). Computerized CBT (Think, Feel, Do) for Depression and Anxiety in Children and Adolescents: Outcomes and Feedback from a Pilot Randomized Controlled Trial. Behavioural and Cognitive Psychotherapy, 39(03), 273-284. https://doi.org/10.1017/S135246581000086X

Stewart, R. W., Orengo-Aguayo, R., Young, J., Wallace, M. M., Cohen, J. A., Mannarino, A. P., \& De Arellano, M. A. (2020). Feasibility and effectiveness of a telehealth service delivery model for treating childhood posttraumatic stress: A community-based, open pilot trial of trauma-focused cognitive-behavioral therapy. Journal of Psychotherapy Integration, 30(2), 274-289. https://doi.org/10.1037/int0000225

Tarragona, M. (2013). Psicología positiva y terapias constructivas: Una propuesta integradora. Terapia psicológica. 31. 115-125. https://doi.org/10.4067/S071848082013000100011

Valenzuela, M., Ibarra, R., Zubarew, G., y Loreto, C. (2013). Prevención de conductas de riesgo en el Adolescente: Rol de familia. Índex Enfermería, 22 (1-2), 50- 54. https://doi. org/10.4321/S1132-12962013000100011

Wang, C., Pan, R., Wan, X., Tan, Y., Xu, L., Ho, C. S., \& Ho, R. C. (2020). Immediate psychological responses and associated factors during the initial stage of the 2019 coronavirus disease (COVID-19) epidemic among the general population in China. International journal of environmental research and public health, 17(5), 1729.

https://doi.org/10.3390/ijerph17051729

Whittaker, R., Merry, S., Stasiak, K., McDowell, H., Doherty, I., Shepherd, M., Dorey, E., Parag, V., Ameratunga, S., y Rodgers, A. (2012). MEMO-A Mobile Phone Depression Prevention Intervention for Adolescents: Development Process and Postprogram Findings on Acceptability From a Randomized Controlled Trial. J Med Internet Res, 14(1). https://doi.org/10.2196/jmir.1857 